GUEST EDITORIAL

\title{
Health and rehabilitation sciences in a clinical context
}

Rehabilitation science is an interdisciplinary field that focuses on human function, health and disability. It is a health strategy applied by professionals in the health sector and across other sectors that aims to enable people with health conditions who experience or are likely to experience disability to achieve and maintain optimal functioning in their interaction with the environment. Basic and applied research from health sciences, social sciences, engineering and related fields in the rehabilitation sciences is directed towards: (i) enhancing physical and psychosocial functioning, participation in life situations, and health-related quality of life of persons with ill-health and disabilities; and (ii) informing relevant social and healthcare policy. The basis for the successful development of rehabilitation practice and research is a conceptually sound description of rehabilitation, understood as a health strategy based on a universally accepted conceptual model and taxonomy of human functioning. The International Classification of Functioning, Disability and Health (ICF $)^{[1]}$ provides such a universally accepted conceptual model of understanding of prevention, management and rehabilitation.

Traditionally, the rehabilitation sciences are thought to comprise three key domains: physiotherapy, occupational therapy, and speechlanguage pathology and audiology. However, rehabilitation science is a wide-ranging discipline and also includes physical medicine and rehabilitation, rehabilitation engineering, rehabilitation nursing, assistive technology, rehabilitation counselling, disability studies, orthotics and prosthetics. While rehabilitation-related research is an integral part of informing practice in these different fields, research and practice are cross-cutting and are conducted within a variety of disciplines. Each of the separate existing disciplines are complementary and have distinct perspectives on disability and rehabilitation; yet, they address the 'enabling-disabling' process as a fundamental concept.

\section{Physiotherapy}

Physiotherapy is the science of preventing, correcting, alleviating and limiting physical disability. Physiotherapists work in the health spheres of promotion, prevention, intervention, habilitation and rehabilitation, and develop, maintain and restore people's maximum movement and functional ability, thereby maximising independence and quality of life. Physiotherapists may intervene in children and older persons, and in those affected by injury, pain, disease or environmental factors. Physiotherapy includes the following core activities: (i) examining and evaluating individuals with impairment, functional limitation and disability or other health-related conditions that impair quality of life to determine diagnosis, prognosis and appropriate intervention. Tests and measures used can include assessment of functional capabilities in self-care and home management, and in work, community and leisure activities; balance and locomotion abilities; musculoskeletal, neuromuscular, cardiopulmonary and integumentary systems; sensory and neurophysiological functions (e.g. electromyographic and motor nerve conduction testing); pain; need for and use of assistive, adaptive, orthotic, protective, supportive or prosthetic devices; and environmental barriers; (ii) alleviating impairment, functional limitation and disability by designating, implementing and modifying therapeutic interventions that may include patient-related instruction; therapeutic exercise; functional training in self-care and home management, and in community, work and leisure activities, including instrumental activities of daily living; manual therapy techniques, including mobilisation and manipulation; prescription, application and, as appropriate, fabrication of assistive, adaptive, orthotic, protective, supportive or prosthetic devices and equipment; airway-clearance techniques; wound management; and electrotherapeutic, physical and mechanical modalities; and (iii) preventing injury, impairment, functional limitation and disability, including the promotion and maintenance of fitness, health and quality of life in populations of all ages.

Physiotherapists use skilled evaluation and hands-on therapy, such as mobilisation, manipulation, massage and acupressure; individually designed exercise programmes, relaxation techniques, sophisticated equipment, hydrotherapy and biofeedback, specialised electrotherapy equipment, heat, ice and traction to relieve pain and assist healing and recovery, suitable walking aids, splints and appliances, and patient education.

\section{Occupational therapy}

Occupational therapy uses selected tasks and activities to restore, reinforce and enhance performance in people with disabling conditions by facilitating learning of skills and functions essential to help them to adapt and achieve the capacity to perform with satisfaction to self and others those tasks and roles essential to productive living and to the mastery of self and the environment. Occupational therapists work alongside people of all ages to help to restore functional ability. Unlike physiotherapy, occupational therapy is not directly involved in treating the injury, limitation or disability; rather, the focus of occupational therapy is on developing, enabling and empowering partnerships with clients so that they are able to independently perform tasks or activities that are essential for survival and meaningful to them in the context of their daily lives. Occupational therapists also recommend equipment and assistive devices that increase skills through a programme of therapy.

They serve diverse groups of people in a variety of settings, such as hospitals and clinics, rehabilitation facilities, long-term care facilities, extended-care facilities, industry-sheltered workshops, schools and camps, private homes and community agencies. Occupational therapists receive and make referrals to appropriate health, educational or medical specialists.

\section{Differences between physiotherapy and occupational therapy}

Because professional boundaries between physiotherapy and occupational therapy overlap, many colleagues in the health professions are unclear regarding the distinction between the two fields. Indeed, there are many similarities between the two professions: (i) both disciplines are committed to providing appropriate highquality care and service to clients and share a knowledge base that includes anatomy, physiology, medical sciences, human growth and development, biomechanics, ergonomics, kinesiology, research methods, statistics, communication and interviewing techniques and clinical conditions; (ii) both have well-established working relationships with other healthcare professionals, particularly the medical and nursing professions; (iii) they share similar clinical 
skills in terms of evaluation protocols, interpretation of standardised assessments, reasoning, writing of assessment reports, and similar technical or medical terminology and communication skills; (iv) they work with common diagnostic groups, including paediatrics, hand rehabilitation, work-hardening/vocational rehabilitation, geriatrics, neurology, burns, plastics, rheumatology and orthopaedics, and have a common goal for client intervention, i.e. maximising the client's level of functional independence; $(v)$ occupational therapists and physiotherapists are employed in similar work settings, including acute-care hospitals, rehabilitation centres, outpatient clinics, corporate and industrial health centres, long-term care facilities, home-care agencies, universities and schools; and (vi) there are multiple therapeutic approaches to practice that are common to both professions, including neurodevelopmental therapy/the Bobath approach, sensory integration therapy, craniosacral therapy, Feldenkrais training, myofascial therapy, the Brunnstrom approach to therapy, the Rood approach to therapy and proprioceptive neuromuscular facilitation. ${ }^{[2]}$

However, a clear understanding of professional boundaries and a mutual respect for the skills and abilities of other healthcare professionals enhance professional working relationships. There are fundamental differences between physiotherapy and occupational therapy: (i) the former is concerned primarily with physical interventions that improve function, while the latter is focused on assessment of physical, emotional, behavioural and social needs and adaptations of tasks and roles essential to independence, productive living and the mastery of self and the environment; (ii) a distinction often made in the literature is that occupational therapy is concerned with the upper extremity, while physiotherapy focuses on the trunk and lower limbs; (iii) although perhaps a gross generalisation, occupational therapists often work in the field of mental health, while, typically, physiotherapists do not; and (iv) the distinctions in occupational therapy curricula include psychology, sociology and psychiatry, while those in physiotherapy include human growth and development, biomechanics, movement studies and pathology. ${ }^{[2]}$

\section{Speech-language pathology and audiology}

Speech and language therapists treat babies, children and adults with varying levels of speech, language and communication problems or difficulties with swallowing, drinking or eating. They often deal with a diverse client group, including people with physical and learning disabilities, hearing loss/deafness, psychiatric disorders or dementia, and can treat a range of conditions, including cleft palate, stammering, language delay and voice disorders. Audiology is the science that studies hearing, balance and related disorders. Audiologists diagnose and treat people with hearing loss and proactively prevent related damage through employment of various testing strategies (e.g. hearing tests, otoacoustic emission measurements, videonystagmography and electrophysiological tests). Audiology aims to determine whether someone can hear within the normal range, and if not, which portions of hearing (high, middle or low frequencies) are affected, to what degree, and where the lesion causing the hearing loss is found (outer ear, middle ear, inner ear, auditory nerve and/ or central nervous system). If an audiologist determines the presence of hearing loss or that a vestibular abnormality is present, he or she will provide recommendations to a patient as to what options (e.g. hearing aid, cochlear implant, appropriate medical referral) may be of assistance. In addition to testing hearing, audiologists also work with a wide range of clientele in rehabilitation (individuals with tinnitus, auditory-processing disorders, cochlear implant users and/or hearing aid users), from paediatric populations to older persons, and may perform assessment of tinnitus and the vestibular system. Speech and language therapists and audiologists work as part of a multidisciplinary team alongside other health professionals, and often liaise with family, carers or teachers when developing treatment plans.

\section{Prosthetics and orthotics}

An orthotist provides care to patients with congenital or traumatic disabling conditions of the musculoskeletal structure of the body by evaluating, designing, fabricating, fitting and aligning braces known an orthoses. A prosthetist also provides care to patients with a partial or total absence of a limb by evaluating, designing, fabricating, fitting and aligning artificial limbs known as prostheses. ${ }^{[3]}$ The work of orthotists and prosthetists occurs in one of five domains: clinical assessment, patient management, technical implementation, practice management and professional responsibility; the work is undertaken in close collaboration with colleagues in physiotherapy, occupational therapy and engineering.

\section{Disability studies}

Disability is an academic discipline that examines the meaning, nature and consequences of disability. Initially, the field focused on the division between impairment and disability, where impairment referred to a physical impairment in an individual's body or mind, while disability was considered a social construct. There are two distinct models of disability: social and medical. The distinction between the two models is blurred, increasingly so with greater focus on interdisciplinary research. Disability studies include disability history, theory, legislation, policy, ethics and the arts, and the focus is on the lived experiences of individuals with disabilities in practical terms. A key focus of work regarding disability is that individuals with disabilities should have greater access to civil rights and improved quality of life.

\section{Scope of work}

Professionals in the health and rehabilitation sciences have a wide choice of work situations that encompasses their entire life course: from teaching a young mother in preparation for the birth of her child to handling babies with lung infections or cerebral palsy, to assisting older children and adults to become mobile again after surgery, injury or burns, and to improving the quality of life of those in old-age homes and helping them to retain and regain their independence. As such, professionals in the rehabilitation sciences work in public and private hospitals, private practice, community health centres, day-care centres, nursing homes, sports centres and with sporting teams, schools and preschools, academic and research centres, universities and training institutions, specialised physiotherapy, occupational, speech and language therapy, orthotic and audiology institutes, health policy development units and special centres for people with physical disabilities. Importantly, the nature of the work is wide ranging and includes engagement in consultation, education and research.

\section{Health and rehabilitation and disability in South Africa}

The prevalence of disability in South Africa (SA) is contentious in part because stakeholders are not agreed on a definition of disability. Current definitions are applied in terms of different legislation and contexts, and focus primarily on impairment, without necessarily addressing the contexts in which barriers limit participation. ${ }^{[4]} \mathrm{A}$ major limitation in determining true levels of disability in SA is that disability prevalence surveys are usually based on reported disability, 
often by a proxy informant, which may over- or underestimate the true prevalence. ${ }^{[4]}$

According to Statistics SA (StatsSA), based on census 2011 data, ${ }^{[5]}$ the national disability prevalence rate is $7.5 \%$, being more prevalent among females than males ( $8.3 \%$ and $6.5 \%$, respectively). This report, however, excluded children $<5$ years of age and people with psychosocial and certain neurological disabilities. In SA, 11\% of people $>5$ years old had visual difficulties, $4.2 \%$ had cognitive difficulties, $3.6 \%$ had hearing difficulties and $2 \%$ had communication, self-care and walking difficulties. Provinces with the highest reported disability were the Free State and Northern Cape (both 11.0\%). Reported disability figures for the remaining provinces, in descending order, are North West (10.0\%), Eastern Cape (9.6\%), KwaZulu-Natal (8.4\%), Mpumalanga (7.0\%), Limpopo (6.9\%), Western Cape (5.4\%) and Gauteng (5.3\%).

The overall prevalence of childhood disability in SA has been studied over the past 30 years, but recent data are scant. ${ }^{[6]}$ Moreover, estimates of child disability prevalence are not directly comparable, as studies use different definitions of disability and methods of data collection. StatsSA estimates that the SA population $>60$ years of age is $7.8 \%$ and the proportion of people with disabilities in the $60-69$-year age group is $14.5 \%$, rising to $34.7 \%$ in the $>70$-year group. ${ }^{[7]}$ Population ageing is associated with impaired functioning and mobility limitations (e.g. impaired vision, glaucoma, diabetic retinopathy, hearing loss, and impaired mobility due to strokes, falls and bone and joint conditions) ${ }^{[8]}$ The prevalence of Alzheimer's and Parkinson's diseases and dementia in SA is unknown. ${ }^{[9]}$ Decreased childhood mortality in SA has had the unintended and unfortunate consequence of increased childhood disability, as is evidenced by the number of children presenting with developmental delays and cerebral palsy. ${ }^{[10]}$

The role of disability in entrenching and exacerbating the cycle of ill-health and poverty is often inadequately understood in health service planning. In 2015, the Department of Health launched the Framework and Strategy for Disability and Rehabilitation Services in South Africa 2015 - 2020, ${ }^{[4]}$ which outlines comprehensive and integrated disability and rehabilitation services within the broader health and developmental context to facilitate improved access at all levels of healthcare. With improved treatment and support, people live longer, resulting in a higher number of people experiencing chronic conditions/illness and disability, which places a larger burden on household and fiscal finances, facilities and human resources in the country.

In $\mathrm{SA}$, the health and rehabilitation sciences are well established in clinical practice, both within the public and private health sectors and in most of the faculties of health sciences that offer underand postgraduate qualifications in these disciplines. However, after promulgation of a plethora of progressive health policies since 1994, the SA public health system reflects a number of stumbling blocks in relation to implementation. Rehabilitation professionals are not sufficiently equipped or allowed the opportunity to comprehensively implement the primary healthcare (PHC) philosophy and principles from a bottom-up approach. Training with regard to social health determinants and their impact on ill-health and health outcomes is insufficient. The inadequate understanding of the advocacy role that rehabilitation professionals could play in addressing social health determinants, remains a challenge in healthcare. Rehabilitation, a pillar of PHC, is poorly understood in terms of its role within the SA health system. ${ }^{[11]}$ Reflecting on the opportunities and challenges faced by rehabilitation community service therapists in the delivery of rehabilitation services in a rural area of the Eastern Cape Province of SA, Ned et al. ${ }^{[11]}$ argued for the development of rehabilitation as a vehicle for addressing social determinants of health, with community service practitioners playing a critical role in addressing the inequities within the healthcare package, and highlighted existing gaps in the delivery of rehabilitation services in SA. The high crime rates in the country also hamper efforts regarding community and individual rehabilitation.

This issue of CME includes two interesting reviews. The first article, by Naidoo et al. ${ }^{[12]}$ describes the opportunities for physiotherapy to be applied to rehabilitation and prohabilitation across the lifespan in dealing with non-communicable diseases (NCDs). The authors argue strongly that while overweight/obesity rates and poor nutrition are significant preventable causes of NCDs in SA, physiotherapy may play an important role by promoting physical activity, physical function and maintenance of health, and by maximising health-related quality of life in the spheres of promotion, prevention, treatment, habilitation and rehabilitation across the lifespan. In the second article, Ramma et al. ${ }^{[13]}$ provide an update on ototoxic drugs, mechanisms of ototoxicity and strategies for the prevention of treatment-induced ototoxicity. They remind us that the focus of treating physicians should be as much on saving lives as it is on preserving hearing and vestibular function to maintain social integration and quality of life of patients.

\section{Ntobeko A B Ntusi}

Division of Cardiology, Department of Medicine, Faculty of Health Sciences,

University of Cape Town and Groote Schuur Hospital; Cape Universities Body Imaging Centre, Faculty of Health Sciences, University of Cape Town; and Hatter Institute for Cardiovascular Research in Africa, Department of Medicine, Faculty of Health Sciences, University of Cape Town, South Africa ntobeko.ntusi@uct.ac.za

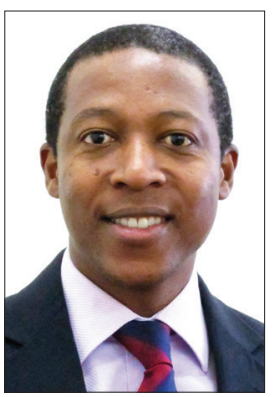

1. World Health Organization. International Classification of Functioning, Disability and Health (ICF) Geneva: WHO, 2002.

Brown GT, Greenwood J Occupational therapy and physiotherapy: Similar, but separate. Br I Occup The 1999;62(4):163-170.

Brandt EN Jr, Pope AM, eds. Enabling America: Assessing the Role of Rehabilitation Science and Engineering. Washington, DC: National Academies Press, 1997.

Engineering. Washington, DC: National Academies Press, 1997.
Department of Health. Framework and Strategy for Disbaility and Rehabilitation Services in South 4. Department of Health. Framework and
Africa 2015 - 2020. Pretoria: DoH, 2015.

5. Statistics South Africa. Census 2011: Profile of Persons With Disabilities in South Africa. Report No. 03-01-59. Pretoria: StatsSA, 2014.

6. Department of Social Development, Department of Women, Children and People with Disabilities, South Africa, and United Nations Children's Fund. Children with Disabilities in South Africa: A Situation Analysis 2001 - 2011. Pretoria: DSD, DWCPD and UNICEF, 2012.

7. Statistics South Africa. Mid-year Population Estimates Report. Pretoria: StatsSA, 2013

8. Joubert J, Bradshaw D. Population ageing and its health challenges in South Africa. In: Steyn K, Fourie J, Temple N, eds. Chronic Diseases of Lifestyle in South Africa 1995 - 2005. Cape Town: South African Medical Research Council, 2006.

9. Dementia, the Invisible Disability. What are we up against? The DG Murray Trust Hands-on Learning Brief 73, 2014:1-3. http://www.dgmt-community.co.za/organisations/dementia-sa/learning-briefs/raisingBrief 73, 2014:1-3. http://www.dgmt-community.co.za/organisations/den
awareness-about-dementia-south-africa (accessed 25 January 2019).

10. Chhagan MK, Kauchali S. Child development and developmental disability. Paper presented at Developmental Disabilities Research Collaborative (DDRC) between the University of KwaZulu-Natal (UKZN), Department of Health, Department of Education and Department of Social Development, (UKZN), Department of Health, Department of Education and Department of Social Development, Durban, 28 March 2011. Durban: University of KwaZulu-Natal, 2011. http://www.ruralrehab.co.zad
uploads/3/0/9/0/3090989/children_development_and_developmental_disabilities_ddrc_2011.pdf (accessed 25 January 2019).

11. Ned L, Cloete L, Mji G. The experiences and challenges faced by rehabilitation community service therapists within the South African primary healthcare health system. Afr J Disabil 2017;6:311. https:// doi.org/10.4102/ajod.v6i0.311

12. Naidoo N, Barnes R, Mlenzana N, Mostert K, Amosun SL. Physiotherapy in rehabilitation and prohabilitation across the lifespan. S Afr Med J 2019;109(3):142-144. https://doi.org/10.7196/ SAMJ.2019.v109i3.13902

13. Ramma L, Schellack N, Heinze B. Prevention of treatment-induced ototoxicity: An update for clinicians. S Afr Med J 2019;109(3):145-149. https://doi.org/10.7196/SAMJ.2019.v109i3.13903 